\title{
A Review of Self Decision Making Applications
}

\author{
A.A. Obiniyi \\ Department of Mathematics, \\ Ahmadu Bello University Zaria, Kaduna, \\ Nigeria.
}

\author{
O N Oyelade \\ Department of Mathematics, \\ Ahmadu Bello University Zaria, Kaduna, Nigeria.
}

\begin{abstract}
Intelligent agents have been employed in the field of artificial intelligence to develop their cognitive capability so as to populate their knowledge base and as well carry out tasks that proves their ability to carry act or respond to stimuli just like a human would have done. Furthermore, agents with different functionalities that may be harmonized to render a given service are been correlated together to form multi-agent. However, this multi-agent systems are been further categorized into those that carry out decisions and those that carry out simulation. This paper researches into decision making applications (also called decision support systemsDSS) that are driven by semantic web technologies, and also looks into their common components and functionalities. Then it points out the prospect that awaits future decision support systems with respect to the growing research in the semantic web technologies.
\end{abstract}

\section{Keywords:}

DSS, Agents, and Semantic Web.

\section{Background to the Study}

A decision support system (DSS) is an integrated set of computer tools allowing a decision maker to interact directly with computer to retrieve information useful in semi structured and unstructured decisions. DSS is also an interactive computer system that is easily accessible to, and operated by computer amateur who uses it to plan and as well make decision (Ismail, 2011). Also, DSS could be defined by Misdolea, (2010) as a class of information system which includes but not limited to computerized systems- and they provide support for business and organizational decisionmaking activities. Decision support systems are also known as decision making system. Basically just as pointed out in the definitions above, they provide intelligent and precise information that will support decisions on every occasion. However, DSS is built upon several components that which include: knowledge base, reasoning faculties, interactive interfaces (Mostly graphical user interface) both for input and output.

DSS tools deals with all the characteristics of information system which are; Access to knowledge, communication of knowledge, use of knowledge, representation of knowledge on all organization (Misdolea, 2010). So, it may be safe to say that DSS are information systems. Decision, making systems interfaces with humans in providing the necessary functionalities it possess to user. Hence this is why most DSS are often designed with three basic human behaviors which takes part in the decision making process. These behaviors are; the first is cognition, activities of the mind associated with knowledge, the second is the action of mind that deals with emotion, feeling, mood and temperament (Ismail, 2011).
Decision making systems are intelligent agents that can make intelligible decisions that humans can take in any circumstance. Some underlying reasoning technologies employed in archiving this are Rule Based Systems, Neural networks, Fuzzy cognitive maps and Fuzzy Rule based specifications or Clustering algorithm. Each of this reasoning schemes are employed based on the type of underlying data model and as well level of reliability demanded from the result the DSS provides. Rule based systems and fuzzy rulebased classification use exact matching on rules built on mature and established domain knowledge, whereas, neural networks approach do not provide justification for the resulting knowledge (Paul, 2011). However, this paper aims at focusing on both the data modeling and inference making using the Semantic Web technologies.

\subsection{Decision Support System and Semantic Web}

Semantic web initiative is meant to provide machine-tomachine communication. This intention was well captured in the vision of the author Tim Berners-Lee which says "Evolution of objects from being principally human-readable documents to contain more machine-oriented semantic information" (Berners-Lee et al.,1994). A 'Semantic Web', which trade, bureaucracy and our daily lives will be handled by machines talking to machines. The 'intelligent agents' people have touted for ages will finally materializes ". Research in the development of tomorrow's web is focused on the exchange of not only documents, but on the exchange of data and services, human-to-machine communication and machine-to-machine communication (Casey et al, 2002).

The concept of decision support systems evolved from two research areas: theoretical studies of organizational decision making between 1950s and early 1960s. And the technical work of on computer systems. The first type of such systems was the executive information systems (EIS), group decision support systems (GDSS) and organizational decision support systems (ODSS), all evolved from the user and single modeloriented DSS. Subsequently online analytical processing (OLAP) broadened DSS before new web based analytical applications were introduced in the new millennium (Okereke, 2010).

In a situation whereby a user uses an open world database (such as Internet), search requests are formed into queries that will syntactically retrieve the needed result for the user. However, in closed world database (such as enterprise) data are modeled in a preconceived pattern and leading to an execution of queries that will return result to the user to also be preconceived. But web-based technologies are having a major impact on the design, development, and implementation of processes for all types of decision support systems (Necula, 2012). Hence, considering the potentials of Semantic web 
technologies, this paper proposes that developing decision making systems that the data is been modeled with some Semantic Web technologies will further enable new and inferred knowledge to be added to the existing knowledge base and as well give convenient support to the reasoning functionality of DSS.

Therefore, this paper is being geared towards reviewing some decision support systems that are built upon Semantic Web technologies.

\subsection{Functionalities of Decision Support System.}

Various decision making systems have been designed or developed and tailored for specific environment. However, there are some basic functionality and benefits that may be identified with virtually all decision making systems. This section is focused at divulging these functionalities and the benefits of DSS.

Most decision making applications perform one or more functions and some of these functions include collection of information to update or enlarge the knowledge base, integration of divers sources of knowledge and as well subsystems, self-driven information publication for decision makers and lastly, an organized and well patterned presentational approach. Moreover, even decision making applications or DSS are coupled up from integrated subsystems. These various subsystems collectively provide the listed functions. All those functions are performed through different types of DSS software which such as;

a. Data Warehouse: This is repository for the store of electronic data.

b. Data Mart: Data store targeted at one or more data store.

c. Data Cube: A three dimensional way for presentation of data.

d. Data Mining: This is a technique for accessing data so as to intelligibly analyze the needed knowledge that will be presented to the user.

e. DataWeb: this serves as repository for data collected over the web.

These types of DSS software fit into the chain of data implementation (Misdolea, 2010), according to its four fundamental functions enumerated in section 1.3.

\subsection{Categories of Decision Support System}

Decision making systems can be categorized into several types, some of which are;

a. Group Decision Support System (GDSS): This is an interactive computer-based system that facilitates users in finding (partial) solutions to semi-structured problem. And its uses a set of decision makers working together as a group (Okereke, 2010). GDSS acts intelligently and co-operatively in a complex domain with potentially high data rates and makes judgments that model the very best human technicians (Taghezout et al, 2009).

b. Spatial Decision Support Systems (SDSS): Is an interactive, computer-based system designed to support a user or group of users in archiving a higher effectiveness of decision making while solving a semi-structured problem (Okereke, 2010). SDSS have emerged from the co-evaluation of classic DSS research in geospatial or information systems (GISs) (Casey et al, 2002).

c. Web-Based Decision Support System (WBDSS): With the development of internet, WBDSS have become a new trend in DSS research to now provide us with some relevant applications that in turn provides tools and knowledge to improve several aspects of human developments like health care (Ogrima et al, 2012). WBDSS are majorly web applications with characteristics of normal DSS.

\section{Decision Making Applications}

The main thrust of this work is to review some decision support systems (DSS) that were built upon the technologies provided by the Semantic Web initiative. Hence, the following discussions in this section are channel towards this study.

Some of the decision making applications to be considered will range in the list of their categories listed in the section 1.4. The first of such application is a research work by Taghezout et al (2009). The research work proposed to model a group decision support system based on multi-agent architecture. The application was developed for a Boiler Combustion Management System. Usually, the human exploiting engineer is supposed to go around checking for breakdown in the system, analyze it, and diagnosing it on local site before contacting other exploiting engineer. However, the research designed a DSS in category of GDSS, which intelligently detect a malfunction in the system, diagnose the defect and then suggest one or more several appropriate cure locations. The system is a multi-agent system that comprises of the following agents; Interface Agents (IA) continuously receives data from the process so as to capture the current state of the system. A Decision Maker and Agent (DMA) receives user delegated task specification from a IA and interprets the specifications and extracts problem solving goals, forms plan to satisfy these goals, identifies information seeking sub-goals that are present in its plan and then decomposes the plan before coordinating the appropriate Information Retrieval Agent (IRA) which primarily provides intelligent information services . A Modeling Agent (MA) anticipates the occurrences of contingencies using mathematical and computational models. Knowledge Management Agent (KMA) comprises, manages, and updates knowledge base. Diagnosis Agent (DA) is activated by the receipt of information from the decision maker and agent (DMA) which indicate that there might be fault. And lastly, the Action Agent (AA) generates a plan of action which can be used to repair the process. Figure 2.1 captures the interaction of these agents. 


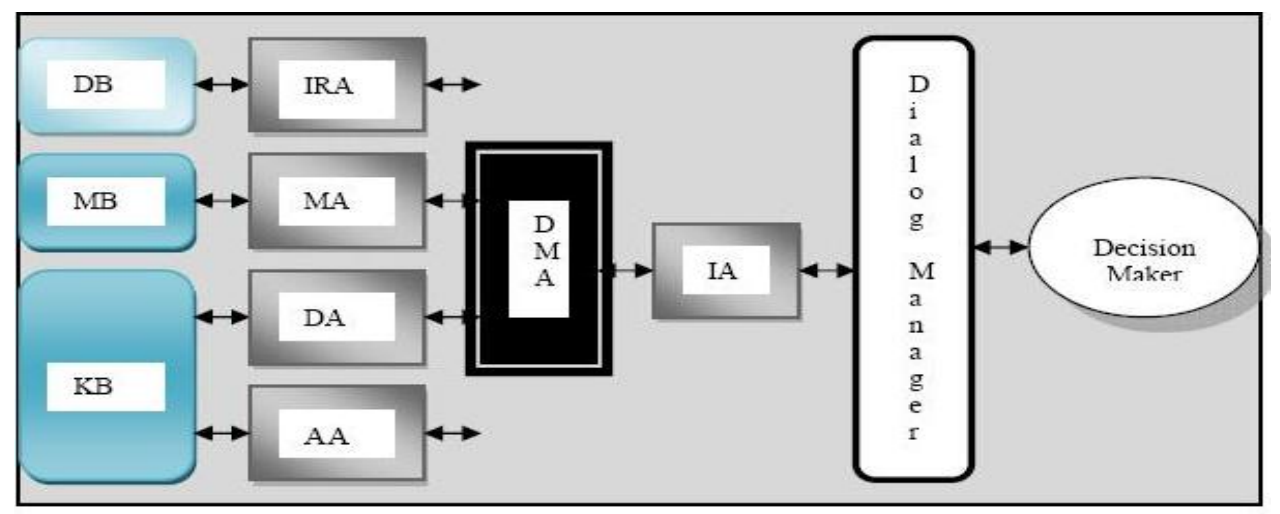

Figure 2.1: Agent architecture for individual DSS (Taghezout et al, 2009)

Casey et al, (2002) in another related research work, proposed a semantic web-enabled navigation and spatial decision support system is been enhanced by semantic methodologies by providing a simple example of tourist trying to navigate from one location to another by relying on and advanced handheld device. The application allows the user type or speaks in his query in the handheld device and this query would be accepted and processed by a geo-location service (agent). Submitted with the tourist query is the geographical coordinates of the tourist current location. Using Semantic based methodologies; the geo-locating agent would begin to break down the submitted query based on ontological references. Having determined the location specified in the query must be located. And this ontology will include both a description about sought for the location and its coordinates.

The agent will then recognize that a database of geospatial information is available for querying. And using the coordinate of the tourist and the coordinate of the destination desired by the tourist, the

shortest route to the destination will be determined by the agent and as well it will simultaneously identify from the same ontology an instance of a maintenance schedule effective from the item of the tourist query. Since the tourist 's interest is not considered to be a map but point-to-point direction for locating a desired place, then the final processing step to be executed by the geo-location agent is the translation of the discovered route into waypoints corresponding to directions along the route. The tourist then receives the waypoints on their handheld device. These waypoints provide $s$ turn-by-turn directions to the desired destination. Semantic Web methodologies have been employed in assisting the tourist to achieve the following; fist, it provides minimal interface to the GIS/SDSS that would have complicated the human-computer interaction. Secondly, the decision-time reasoning has been reduced by the agent. Lastly, disaggregation of spatial data which resulted from rendering only the turn-by turn waypoints to the tourist, instead of a webpage or other document type presentation format was also added.
Ogrima et al. (2012) also researched into an aspect of DSS application that can be used in the prescription of herbal medicine. This DSS application was implemented in a webbased format. The user is provided with an interface that displays the ailments, symptoms, medication, and prescription for each corresponding ailments. The system administrator also has his own interface that includes the buttons that is necessary for updating the database. The database stores the newly discovered ailment and their likely symptoms and cure. The components of the system are as follows;

a. Internet Terminals/Devices: this helps the user to connect to the dedicated Internet devices connected to the server where the application resides.

b. User Interface: enables users to enter information that will be used to carry out diagnosis. This will then be used to access the knowledge base.

c. Web Server: Servers as the gateway application that enables user and the application to send/receive messages.

d. Firewall: Checks information coming from the Internet or network and then either block or permits its passage.

e. Knowledge Base: consists of some encoding of the domain of expertise for the system which can be in the form of knowledge modeled with Semantic Web languages, procedural representation, production rules and frames.

f. Inference Engine: controls and coordinates the dialog that ensues between the system and the user through the user interface.

g. Database: is a fundamental part of the system and it is also called the working storage. This works hand in hand with the knowledge base and the inference engine.

The architecture of this system is shown in Figure 2.2 


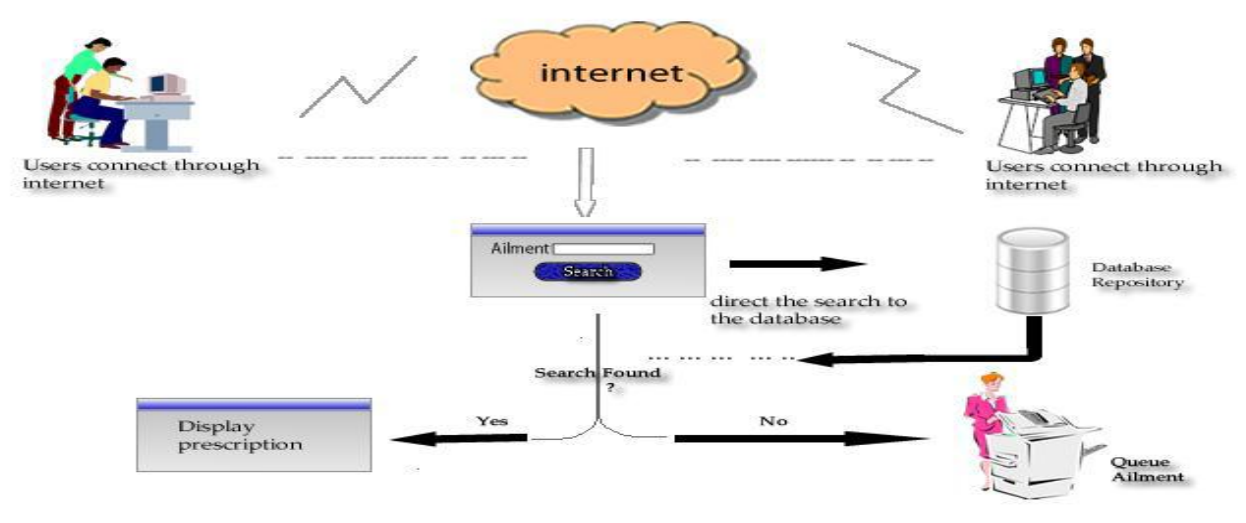

Figure 2.2: Client server operation of Herbal Medicine Prescription DSS (Ogrima et al, 2012).

DSS application that fully based on Semantic Web technologies usually demonstrate a great form of genius that human is expected to exude. One of such application is a research work to be reviewed here is Skeletal Dysplasia DSS (Paul, 2011). The research argues that Semantic Web technologies can be employed in providing the appropriate means for encoding knowledge and enable reasoning complex forms of reasoning in a skeletal dysplasia. Skeletal dysplasia comprises of a group of genetic disease characterized by highly complex, heterogeneous and sparse data. The crux of the application developed by this research was to develop a decision support methods in the skeletal dysplasia domain by this research was to develop a decision support methods in the skeletal dysplasia domain by applying uncertainty reasoning over Semantic Web. One of the challenges of current decision support systems that are not built upon Semantic Web technologies is the task of interoperability of knowledge that is sourced from different places; hence, the research work tackled this challenge by using some Semantic Web technologies. The decision making application developed was via an ontology-based interoperable framework tailored towards the skeletal dysplasia domain. The research combines ontology techniques with inductive statically reasoning techniques. This was integrated within SKELETOME community-driven knowledge curative platform.

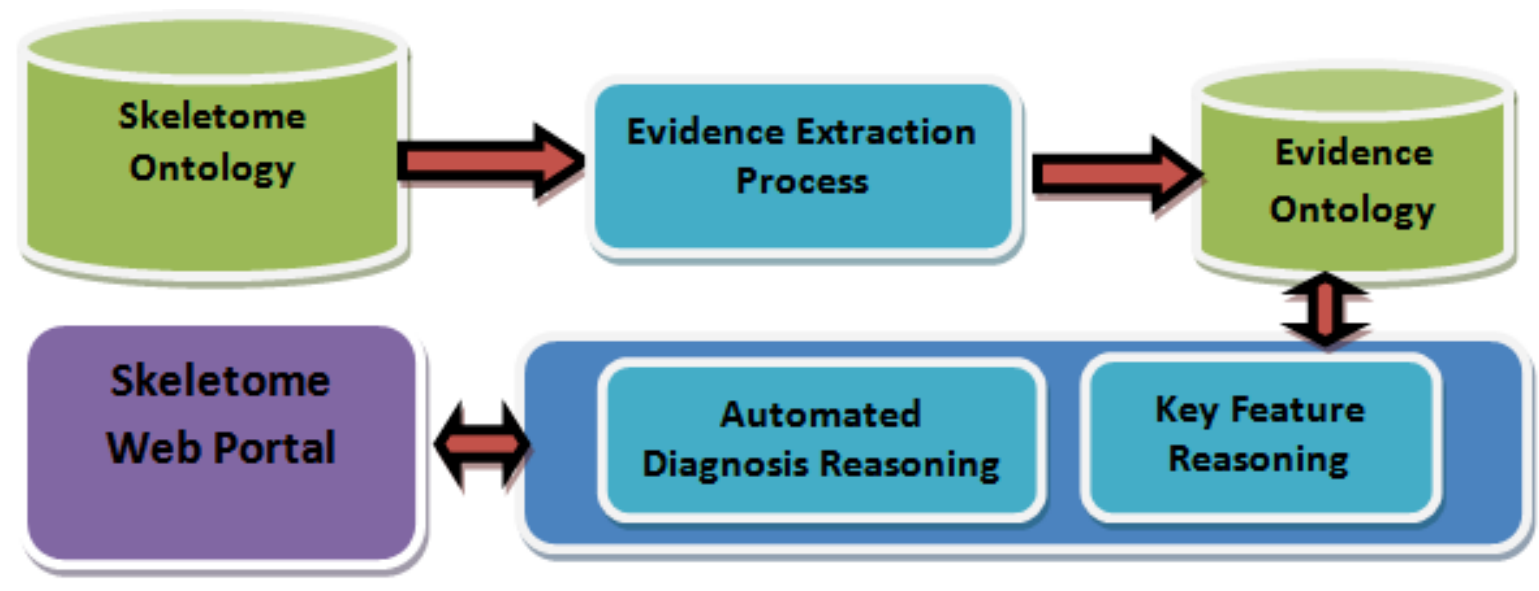

Figure 2.3: System Architecture of Skeletal Dysplasia DSS (Paul, 2011).

Figure 2.3 reveals that the SKELETOME ontology, which was developed to store the essential knowledge of skeletal dysplasia domain, is a fundamental prerequisite of the framework. It captures past and newly emerging patient cases while Evidence Ontology stores generalized evidences. The evidence extraction process induces generalized evidences from the exiting patient cases and encodes the resulting knowledge in the Evidence Ontology by taking account of the lack of mature domain knowledge. Furthermore, as reasoning is been carried out over the induced generalized evidences enables the development of the targeted decision support methods.

Some challenges with the existing DSS are associated with the issue of maintaining and building up on the facts and rules that enable the system to be able to intelligently take necessary decision per time. Some DSS were developed solely on high level programming languages, thereby resting on the control structures in realizing the intelligence aspect of the system. While some other category of DSS were built 
upon mathematical models which to some extent may not be completely implementable by the programmer. Furthermore, some of these DSS have been improved upon based on the use of logic programing languages like Prolog and Lisp. However, it should be noted that the Semantic Web provides interoperable technologies, such as data modeling languages (Web Ontology Language -OWL), query languages (Semantic Query-enhanced Web Rule Language -SQWRL) and rule languages (Semantic Web Rule Language SWRL) that can be employed in deploying an efficient functionality for both the facts and rules that drives the DSS.

\section{Prospects of Future Decision Support Systems.}

In this paper, some decision making applications have been reviewed, however, Taghezout et al (2009) reviewed in section 2.1 does not make use of Semantic web technologies, though it demonstrate a form or category of DSS that ought to be considered. Some parameters that should guide one in evaluating these applications are; Decision making effectiveness, performance, ease of understanding and lastly, scalability. The first parameter consists of some other parameters that can be used to measure it, and these are Accuracy, Precision and Recall. They are metrics that can be used to access effectiveness (Paul, 2011). Reasoning and inference making are easily done by exploiting some Semantic Web technologies. Rule languages (such as Semantic Web Rule Language, SWRL) and rule systems are widely used in diagnosis, business fact finding, compliance monitoring, and process control applications (O'Connor, 2005). Parsia et al (2005) pointed out that people attach some pragmatic features to rules and that they mesh better with our own way of thinking, recalling, and reasoning about certain problems. Hence, the Recall metric - which is one of the features of human intelligence that makes decision making applications intelligent- can be well implemented using rule languages that are obtainable from Semantic Web languages or technologies. For instance, when a diagnostic application can recall the previous diagnosis carried out on a patient, then such application can be said to be effective. Also, being precise and accurate are some indications of human expertise that brings about reliance on human to manually manipulate a system, however, resting on some rule engines like Jess, then precise and accurate rules can be implemented in a decision support systems so as to add up accuracy and preciseness to the entire DSS. The other three parameters; Performance, ease of understanding and scalability depends majorly on the design on interface design and some form of software engineering techniques employed in the design of DSS application.

El Sawy (2010) in a presentation pointed out several prospects of future DSS. And some of them includes improving DSS applications to handicapped persons so that faculties that argument what the handicapped do not have are built into such DSS, value and intelligent latency ought to be reduced in DSS. Other forecasted development in DSS application also are, issue-based DSS application, deeper understanding of decision making and scanning, ability to search for a semantic entity-relationship model and lastly, managing distractions and interruptions in DSS.

\section{Summary}

There are several automations of manual systems that cannot be tied to DSS or intelligent applications. Having reviewed some decision making applications, one thing that is paramount with them is the ability to take input from user and take intelligent decisions based on a information or knowledge base and rule sets that will enhance reasoning. So then when a manual system is been automated, the gains of such automation would be enormous and more appreciable if there is an element of self-decision making capability is added into it. The work of Paul (2011) reviewed in this work demonstrates the use of Semantic Web technologies in implementing the form of a DSS into the application revealing that the Semantic Web technologies have the power to actualize such intelligent functionalities into any desired DSS. Therefore, it behoove developers of DSS to tap into several available knowledge representation languages, rule engines, query languages and reasoning engines that are found in Semantic Web technologies in other to realize a selfdecision making application they desire.

\section{Conclusion}

This research has been focused on reviewing some DSS applications that are built upon the Semantic Web initiative. This influenced the choice of some of the DSS applications reviewed in the work. The multi-agent boiler combustion management system actually is not Semantic Web oriented, but it was included to exemplify the category of DSS called GDSS. The second reviewed work is majorly a Semantic Web based DSS application. Here, the navigation and spatial decision support system's information is been modeled ontologically using a ontology language. Thirdly, a webbased herbal medicine prescription DSS was also reviewed where rule was employed in the decision making exercise. Lastly, the skeletal dysplasia reasoning application is able to reason over complex knowledge base (Semantically modeled) in this particular domain and then makes accurate prescription for patient. All these reviewed work and a host of other such DSS applications points to the fact that field of Artificial intelligence can greatly exploit the Semantic Web technologies in a bid to develop intelligent application that aids self-decision making.

In conclusion, with the advent of stronger inference making technologies courtesy of the Semantic Web initiative, developing DSS applications can be made to perform intelligently as human would have performed.

\section{References}

[1] Casey, M. J., and Austin, M. A., (2002). Semantic Web Methodologies for Spatial Decision Support, Institute for Systems Research and Department of Civil and Environmental Engineering, University of Maryland, college Park, USA, pp1.

[2] El Sawy A. O., (2010). Decision Support and Environmental Scanning: Past Hallucinations, Present Realities, and Future Prospective, Marshal School of Business University of Southern California, pp 41.

[3] Ismail, S.T., (2011). The role of Marketing Information System on Decision Making- An Applied Study of Royal Jordanian Airlines (RJA), International Journal of Business Social Science Vol. 2 No. 3, pp 4.

[4] Misdolea, R., (2010). Decision Support System and Customer Relationship Management as Components of the Cybernetic System Enterprise, Informatica Economică Vol. 14, No. 1, pp 3.

[5] Necula, S. B., (2012). Implementing the Main Functionalities Required by Semantic Search in Decision 
Support Systems. INT J COMPUT COMMUN, ISSN 1841-9836 7(5):907-915, pp 908.

[6] O'Connor, M., Knublauch, H., Tu, S., Grosof, B., Dean, M., Grosso W., and Musen, M., (2005). Supporting Rule System Interoperability on the Semantic Web with SWRL. Stanford Medical Informatics, Stanford University of School of Medicine, Stanford, pp 1.

[7] Ogirma, S. A. O., Olatunde, O. S., Olusayo, O. E., Oluwafunke, O. A., (2012). Web Based Decision Support System for Prescription in Herbal Medicine. Transnational Journal of Science and Technology Vol. 2, No. 11.

[8] Okereke G. C., (2010). Decision Support Systems (DSS), National Open University of Nigeria, School of Management Sciences, pp. 24.
[9] Parsia, B., Sirin, E., Cuenca, B. G., Ruckhaus, E., and Hewlett D., (2005). Cautiously Approaching SWRL, Preprint submitted to Elsevier Science, pp 3.

[10] Paul, R., (2011). DC Proposal: Decision Support Methods in Community-Driven Knowledge Curation Platforms, School of ITEE, The University of Queensland, Lucia, Australia, pp 2.

[11] Taghezout, N., Adla, A., and Zaraté, P., (2009). A Multiagent Framework for Group Decision Support System: Application to Boiler Combustion Management System (GLZ). International Journal of Software Engineering and Its Applications, Vol. 3, No. 2.

[12] Tim Berners-Lee (1994). Weaving the Web, The MIT Press Bookstore, Cambridge, pp. 9 